\title{
L'effet du déséquilibre des sexes sur le comportement matrimonial : comparaison entre la Nouvelle-France, Saint-Domingue et la Nouvelle-Orléans
}

\section{Paul Lachance}

Volume 39, numéro 2, automne 1985

Histoire de la famille

URI : https://id.erudit.org/iderudit/304350ar

DOI : https://doi.org/10.7202/304350ar

Aller au sommaire du numéro

Éditeur(s)

Institut d'histoire de l'Amérique française

ISSN

0035-2357 (imprimé)

1492-1383 (numérique)

Découvrir la revue

Citer cet article

Lachance, P. (1985). L'effet du déséquilibre des sexes sur le comportement matrimonial : comparaison entre la Nouvelle-France, Saint-Domingue et la Nouvelle-Orléans. Revue d'histoire de l'Amérique française, 39(2), 211-231.

https://doi.org/10.7202/304350ar d'utilisation que vous pouvez consulter en ligne. 


\title{
L'EFFET DU DÉSÉQUILIBRE DES SEXES SUR LE COMPORTEMENT MATRIMONIAL: COMPARAISON ENTRE LA NOUVELLE-FRANCE, SAINT-DOMINGUE ET LA NOUVELLE-ORLÉANS ${ }^{1}$
}

\author{
PAUL LACHANCE \\ Département d' histoire \\ Université d'Ottawa
}

\section{Introduction}

«Pays de pauvres hommes», «paradis pour femmes» ${ }^{2}$ : la part de vérité de ces aphorismes anglais provient de la rareté des femmes en Amérique coloniale. Dans un article publié en 1945, Herbert Moller a remarqué sous ce rapport le contraste avec le surplus féminin partout en Europe aux $17 \mathrm{e}$ et $18 \mathrm{e}$ siècles ${ }^{3}$. Avant l'établissement d'un équilibre des sexes, un processus qui souvent s'échelonne sur plusieurs générations, le type de mariage dit «européen» - âge au mariage retardé pour les femmes aussi bien que pour les hommes, proportion élevée de personnes qui ne se marient jamais - n'a pas pu se reproduire de l'autre côté de l'Atlantique ${ }^{4}$. Selon Moller, le déséquilibre des sexes dans le Nouveau Monde n'a pas seulement influencé la nuptialité, mais aussi le métissage, les moeurs familiales et la sensibilité religieuse ${ }^{5}$.

Moller se fonde sur le rapport hommes-femmes dans les colonies anglaises, mais l'Amérique française fournit aussi des exemples frappants de déséquilibre des sexes. En Nouvelle-France, le rapport de masculinité a dépassé 200 jusqu'aux années 1660 , avant de tomber à moins de 140 au cours des années 1680 et à moins de 110 à partir de $1715^{6}$. A Saint-Domingue, la colonie préférée des immigrants français au $18 \mathrm{e}$ siècle, le rapport de masculinité de la population blanche n'est jamais descendu au-dessous de 400 et est remonté à plus de 500 dans les deux

\footnotetext{
1 Une première version de cet article fut présentée à la réunion annuelle de l'Institut d'histoire de l'Amérique française, à Québec, le 20 octobre 1984. La recherche fut subventionnée par le Conseil de recherches en sciences humaines du Canada. Je tiens à remercier Jean-Guy Daigle, Chad Gaffield, Yves Landry et Nicole Lemire pour leurs commentaires et suggestions.

2 Jean Meyer, Les Européens et les autres de Cortès à Washington (Paris, Librairie Armand Colin, 1975), 152.

3 Herbert Moller, «Sex Composition and Correlated Culture Patterns of Colonial America», William and Mary Quarterly, 3e série, 2 (1945): 138.

4 J. Hajnal, «European Marriages in Perspective», dans D. V. Glass et D. E. C. Eversley, eds., Population in History (London, Edward Arnold, 1965), 101.

5 H. Moller, op. cit., 129-153.

6 R. Roy et H. Charbonneau, «La nuptialité en situation de déséquilibre des sexes: le Canada du XVIIe siècle», Annales de démographie historique (1978): 287. Voir figure 2.
} 
décennies précédant l'insurrection des esclaves ${ }^{7}$. A la Nouvelle-Orléans, ville principale d'un troisième territoire américain colonisé initialement par la France, le nombre des hommes pour cent femmes dans la population blanche, qui est proche de 100 à la fin de la domination française en 1763 , remonte à plus de 140 sous le régime espagnol et redevient très déséquilibré à la suite de la prise en charge de la Louisiane par les États-Unis en $1803^{8}$.

Notre étude porte principalement sur l'effet du déséquilibre des sexes sur les comportements matrimoniaux à la Nouvelle-Orléans. En premier lieu, à partir des recensements des populations blanche et de couleur libre, nous tracerons l'évolution du rapport de masculinité dans la ville depuis 1791 jusqu'à 1840 . Ensuite, au moyen du modèle du marché matrimonial et à l'aide de comparaisons avec les populations mieux connues de la Nouvelle-France et de Saint-Domingue, nous verrons comment des situations de déséquilibre prononcé ont affecté l'âge au mariage des deux sexes, leurs chances relatives de remariage et la fréquence des mariages exogames. Si les mariages interraciaux étaient rares à la Nouvelle-Orléans, il y avait beaucoup d'intermariages à l'intérieur de la race blanche entre immigrants et femmes du pays, comme en Nouvelle-France au $17 \mathrm{e}$ siècle d'ailleurs ${ }^{9}$. Les mariages entre hommes du pays et fermmes étrangères, par contre, étaient rares; et en général les gens de couleur libres étaient moins exogames que les blancs. Il s'agit donc de savoir si ces variations s'expliquent, au moins en partie, par l'influence de la répartition des sexes sur le choix des partenaires.

\section{La répartition des sexes}

Au début du $19 \mathrm{e}$ siècle la Nouvelle-Orléans est une ville cosmopolite de 8000 habitants $^{10}$. Elle atteint plus de 27000 âmes en 1820 . Trois castes s'y côtoient: les Blancs, les gens de couleur libres et les esclaves. Les Créoles - Louisianais de naissance - forment le noyau de

\footnotetext{
7 Jacques Houdaille, «Quelques données sur la population de Saint-Domingue au XVIIIe siècle», Population, 28,4-5 (juillet-octobre 1973): 863 .

8 En 1763 le rapport de masculinité à la Nouvelle-Orléans chez les hommes de plus de 14 ans et les femmes de plus de 12 ans ne s'élève qu'à 105,4. Le «Précis de recensement général de la Louisiane», mai 1766, AGI, S.D., leg. 2585, indique un rapport de 122,8; le «Resumen general que comprehende todos los Habitantes y Establecimientos de la Colonia de la Luisiana, Hecho el ano de 1766», juin 1766, AGI, S.D., leg. 2595, donne 144,9 hommes pour 100 femmes. D'après le «Resumé général du recensement fait dans la province de la Louisiane, District de la Mobile et Place de Pensacola en 1788», Archives du Ministère des Affaires étrangères (Paris), correspondance consulaire pour la Nouvelle-Orléans, I (1804-1817), f. 55, le rapport de masculinité est 146,4 , à peu près le même qu'en 1766 , si on préfère l'état de la population du mois de juin, et qu'en 1791 comme indiqué dans le tableau 1 de notre étude.

9 Louise Dechêne, Habitants et marchands de Montréal au XVII siècle (Paris, Librairie Plon, 1974), 102-3; Yves Landry et Jacques Légaré, «Le cycle de vie familiale en NouvelleFrance: méthodologie et application à un échantillon», Histoire sociale/Social History, 17 (mai 1984): 11 .

10 George Dargo, Jefferson's Louisiana: Politics and the Clash of Legal Traditions (Cambridge, Harvard University Press, 1975) 6, 179.
} 
la population blanche auquel s'ajoutent des nouveaux venus de langue française de l'Europe et des Antilles françaises, des Américains arrivant de la côte est, et des immigrants de diverses autres nationalités. La majeure partie des gens de couleur libres sont des Créoles, mais ils viennent aussi d'autres États américains, de l'Afrique et surtout de SaintDomingue.

Les dénombrements de la période 1791-1840, qui regroupent la population de la Nouvelle-Orléans par sexe, groupe d'âge, race et statut de libre ou d'esclave, permettent de calculer le nombre d'hommes pour cent femmes adultes parmi les blancs et les gens de couleur libres, les deux castes qui avaient le droit de se marier légalement (tableau 1). On constate tout de suite le contraste entre les deux races et l'ampleur du déséquilibre des sexes. En 1820 le nombre des hommes blancs est plus de deux fois supérieur à celui des femmes blanches et, à partir de 1830 , presque deux fois supérieur, alors que les hommes de couleur ne représentent pas même la moitié des femmes de couleur avant 1830 .

Le rapport de masculinité de la race blanche s'élève entre 1810 et 1820 au même niveau qu'en Nouvelle-France pendant le premier siècle de colonisation ${ }^{11}$. Le déséquilibre inverse des sexes chez les

TABLEAU 1

Le rapport de masculinité par race et année de recensement, paroisse d'Orléans, 1791-1840ª

\begin{tabular}{|lrrrrrcc|}
\hline & \multicolumn{3}{c}{ Population blanche } & \multicolumn{3}{c|}{ Population de couleur } \\
\hline année & hommes & femmes & $\begin{array}{c}\text { rapport } \\
\text { de masc }\end{array}$ & hommes & femmes & $\begin{array}{c}\text { rapport } \\
\text { de masc }^{\mathrm{b}}\end{array}$ \\
\hline 1791 & 792 & 557 & 142,2 & 126 & 343 & 36,7 \\
1805 & 1261 & 963 & 130,9 & 215 & 605 & 35,5 \\
1810 & 3173 & 1995 & 159,0 & - & - & - \\
$1820^{\mathrm{c}}$ & 9165 & 4019 & 230,9 & 1235 & 2773 & 44,5 \\
$1830^{\text {d }}$ & 9244 & 4958 & 186,4 & 1494 & 1824 & 81,9 \\
$1840^{\text {d }}$ & 25791 & 14646 & 180,0 & 3094 & 4859 & 63,7 \\
\hline
\end{tabular}

Sources: Recensement espagnol de la Nouvelle-Orléans, le 6 novembre 1791, MS. dans les archives de la ville de la Nouvelle-Orléans, Bibliothéque municipale; recensement de la Nouvelle-Orléans, le 5 août 1805, comp. par Matthew Flannery (copie imprimée dans New Orleans in 1805: A Directory and $a$ Census [la Nouvelle-Orléans, The Pelican Gallery, Inc., 1936]); liste nominative pour le Territoire d'Orléans, troisième recensement des États-Unis, 1810, MS., folio 295; feuilles récapitulatives pour la Louisiane, quatrième recensement, 1820, 297; cinquième recensement, 1830, 104-105; et sixième recensement, 1840, 256-257.

a'L'unité de recensement utilisé en composant le tableau est la paroisse d'Orléans. Au début du $19^{\mathrm{e}}$ siècle, elle comprend toujours des districts ruraux, mais s'urbanise complètement vers 1840 .

bOn obtient le rapport de masculinité en divisant le nombre d'hommes par le nombre de femmes et en multipliant le résultat par 100. Le tableau tient compte des hommes et des femmes de 16 ans ou plus, sauf pour les recensements de 1830 et 1840 , tel qu'indiqué dans les notes.

${ }^{c}$ Gens de couleur libres de 14 ans ou plus.

${ }^{d}$ Blancs de 15 ans ou plus et gens de couleur libres de 24 ans ou plus. 
gens de couleur libres a aussi un parallèle dans le surplus de femmes dans les populations indienne, noire et cléricale sur l'île de Montréal au 17 e siècle, mais ces groupes-ci sont plus minoritaires que la caste des gens de couleur libres en Louisiane et dans d'autres colonies méridionales ${ }^{12}$. A Saint-Domingue, le surplus des hommes blancs est plus de deux fois supérieur à celui de la Nouvelle-Orléans. Les deux endroits se ressemblent par contre en ce qui a trait à l'excédent des femmes de couleur qui contrebalance largement celui des hommes blancs ${ }^{13}$.

Pour comparer directement le déséquilibre des sexes dans les deux races et mieux suivre l'évolution à travers le temps, il est instructif de confronter les pourcentages du sexe excédentaire dans chaque race, c'est-à-dire ceux ou celles que le déséquilibre dans un contexte de monogamie privait forcément de partenaire conjugal de même race (tableau 2). Le déséquilibre des sexes s'est beaucoup aggravé chez les blancs entre 1805 et 1820 avant de se rétrécir légèrement en 1830 et 1840. Chez les gens de couleur libres il s'atténua à partir de 1805. En 1830 le déséquilibre est pour la première fois nettement moins prononcé de leur côté que du côté des blancs.

Dans la population blanche les variations du rapport de masculinité dans le temps s'expliquent principalement par l'immigra-

TABLEAU 2

Proportion du sexe excédentaire selon la race, la Nouvelle-Orléans, 1791-1840

\begin{tabular}{|ccccc|}
\hline & \multicolumn{2}{c}{ hommes blancs } & \multicolumn{2}{c|}{ femmes de couleur } \\
\hline année & $\mathrm{N}$ & $\%$ & $\mathrm{~N}$ & $\%$ \\
\hline 1791 & 235 & 29,7 & 217 & 63,3 \\
1805 & 298 & 23,6 & 390 & 64,5 \\
1810 & 1178 & 37,1 & - & - \\
1820 & 5146 & 56,1 & 1538 & 55,5 \\
1830 & 4286 & 46,4 & 330 & 18,1 \\
1840 & 11145 & 43,4 & 1765 & 36,3 \\
\hline
\end{tabular}

Source: Tableau 1.

Note: $\mathrm{N}=$ effectifs du sexe excédentaire moins effectifs du sexe déficitaire; $\%=\mathrm{N}$ divisé par effectifs du sexe excédentaire. Par exemple, en 1791, le nombre d'hommes blancs (792) moins le nombre de femmes blanches (557), qui égale 235 hommes en surplus, ou $29,7 \%$ (235/792) de l'ensemble des effectifs masculins de la race blanche.

11 R. Roy et H. Charbonneau, op. cit., 287.

12 Robert Wells, The Population of the British Colonies in America before 1776: A Survey of Census Data (Princeton, Princeton University Press, 1975), 67. Le rapport de masculinité des serviteurs était de 135 à Québec et de 132 à Trois-Rivières, mais il n'y avait que 88 hommes noirs et 86 hommes indiens pour 100 femmes de chaque race et 58 curés pour 100 religieuses.

13 J. Houdaille, op. cit., 863 et 865 . Voir tableaux 2 et 6. 
tion. Poursuivant la stratégie d'utiliser la Louisiane comme province tampon entre le Mexique et les États-Unis, les autorités espagnoles avaient encouragé l'immigration en Louisiane pour déjouer la menace pressentie dans la migration des Américains à travers les Appalaches après la Guerre d'indépendance ${ }^{14}$. A en juger par le déséquilibre des sexes dans la population blanche, moins élevé en 1791 et 1805 que par la suite, on ne devrait pas exagérer le succès de cette politique, du moins dans le milieu urbain. C'est à la suite de l'achat de la Louisiane par les États-Unis que les recensements reflètent un afflux appréciable d'immigrants blancs à la Nouvelle-Orléans. Au début il s'agit surtout d'hommes célibataires, mais à partir de 1830 l'arrivée de familles entières d'Irlandais - avec la croissance naturelle de la population créole - commence à modérer le déséquilibre des sexes ${ }^{15}$.

L'excédent des femmes de couleur libres est la conséquence, d'une part, de la manumission de plus d'esclaves du sexe féminin. Cela est normal, les femmes esclaves étant plus nombreuses en ville que les hommes esclaves ${ }^{16}$. D'autre part, les gens de couleur chez les réfugiés de Saint-Domingue qui se sont rendus à la Nouvelle-Orléans en 1809, en doublant d'un seul coup la population de couleur de la ville, ont aussi renforcé ses structures, y compris la prépondérance féminine ${ }^{17}$.

\section{Modèle et sources}

Pour examiner systématiquement les effets du déséquilibre des sexes à la Nouvelle-Orléans, nous nous servirons du modèle du marché matrimonial. Ce modèle suppose que les représentants de chaque sexe entrent en concurrence pour le meilleur partenaire possible, suivant donc un comportement qui obéit aux lois de l'offre et de la demande et qui est explicable en ces termes ${ }^{18}$. Au cas où l'un ou l'autre des deux

14 Gilbert Din, «Spain's Immigration Policy in Louisiana and the American Penetration, 1792-1803», Southwestern Historical Quarterly, 76 (1973): 256, 271, 274-75.

15 Alan Conway, «New Orleans as a Port of Immigration», Louisiana Studies, 1 (automne 1962): 1-22; Earl F. Niehaus, The Irish in New Orleans, 1800-1860 (Baton Rouge, Louisiana State University Press, 1975), 25.

16 Lawrence Kotlikoff et Anton Rupert, «The Manumission of Slaves in New Orleans, 1827-1846», Southern Studies: an Interdisciplinary Journal of the South, 19 (été 1980): 176. Les femmes sont visées dans $68 \%$ de 1159 pétitions d'émancipation, alors que les esclaves du sexe féminin ne représentent que 58\% de l'ensemble des esclaves en 1830, 1840 et 1850.

17 Le rapport du maire de la Nouvelle-Orléans sur les réfugiés, le 18 janvier 1810 , publié dans Le Moniteur de la Louisiane, le 27 janvier 1810, fait mention de 1377 femmes adultes et de 428 hommes adultes de couleur libre, un rapport à peu près identique à celui de 605 femmes aux 215 hommes de couleur libres selon le recensement de 1805 . Le recensement fédéral de 1810 ne contient pas de renseignements sur l'âge et le sexe des gens de couleur libres, mais il révèle la présence d'un total de 5727 habitants de couleur, enfants compris.

18 Le modèle est analysé par Gary Becker, «A Theory of Marriage», dans The Economic Approach to Human Behaviour (Chicago et Londres, 1976), 206, et par Louis Henry, «Le fonctionnement du marché matrimonial», Jacques Dupâquier et al., éds., Marriage and Remarriage in Populations of the Past/Mariage et remariage dans les populations du passé (London, Academic Press, 1981), 191-98. 
sexes se trouve en surplus, celui qui est moins nombreux devrait être favorisé. Le sexe minoritaire réussirait mieux à trouver les qualités recherchées dans l'autre sexe et serait moins handicapé par des caractéristiques dévalorisantes telles que le veuvage.

Souvent le modèle suppose aussi l'opération de mécanismes de compensation. Le mariage retardé du sexe excédentaire ou le mariage précoce du sexe déficitaire, ou les deux à la fois, auraient comme conséquence de réduire la différence entre les effectifs masculins et féminins sur le marché ${ }^{19}$. Des taux différentiels de célibat pourraient aussi compenser le déséquilibre des sexes, même s'il est impossible d'aborder cet aspect de la question dans notre étude.

Afin d'examiner l'âge au mariage et les possibilités de remariage à la Nouvelle-Orléans, 377 contrats de mariages rédigés entre 1804 et 1819 ont été dépouillés ${ }^{20}$. Un contrat existe pour à peu près un mariage sur quatre parmi ceux qui ont été célébrés dans les églises catholiques au cours de la période en question. Les données de cette source sont plus riches de renseignements que celles qui se trouvent dans les registres des églises. En plus de nous informer sur l'âge, sur l'état civil, et sur les parents des conjoints, $71,8 \%$ des contrats décèlent les fortunes des maris à la veille de leur mariage et $88,6 \%$ le montant et la composition des apports de la femme, ce qui permet une analyse économique aussi bien que démographique du marché matrimonial.

Pour étudier l'effet possible d'un déséquilibre des sexes prononcé sur la sélection des époux, les contrats de mariage présentent l'avantage additionnel de pouvoir mettre les mariages des blancs en parallèle avec ceux des gens de couleur libres. Puisque le code civil de la Louisiane ne permettait pas le mariage interracial, deux sous-marchés matrimoniaux bien séparés coexistaient dans la ville, l'un pour les blancs, l'autre

\footnotetext{
19 Louis Henry, ibid., 193, fournit un exemple théorique du mécanisme de compensation: «Les hommes se mariant plus tard que les femmes et ayant, avant le mariage, une mortalité plus élevée qu'elles, les filles à marier seraient en excédent si elles n'avaient d'autre perspective que d'épouser des célibataires; à désir égal de se marier, le célibat définitif serait alors plus fréquent chez les femmes que chez les hommes. En fait, veufs et veuves se remarient avec des célibataires et l'on sait que le remariage est plus fréquent chez les veufs que chez les veuves; l'effet du déséquilibre des sexes chez les célibataires pourrait donc se trouver compensé, sinon renversé, pour peu que la différence d'âge entre mari et femme ne soit pas trop grande; si, au contraire, elle était très grande, le déséquilibre des sexes serait très accusé chez les célibataires et le nombre de veufs pouvant y remédier se trouverait réduit par la mortalité plus élevée d'hommes nettement plus âgés que leur femme.»

20 Les contrats de mariage sont conservés aux archives notariales du Civil District Court pour la Nouvelle-Orléans. Ils se trouvent éparpillés parmi les actes dans les livres des notaires suivants: Narcissus Broutin (1804-1819), Christoval de Armas (1815-1819), Michel de Armas (1809-1819), Stephen de Quinones (1805-1816), Marc Laffitte (1810-1819), Hugues Lavergne (1819), John Lynd (1805-1819), Pierre Pedesclaux (1804-1816), Philippe Pedesclaux (1817-1819), Carlisle Pollock (1817-1819), et Benedicte van Pradelles (1806-1808). Charles R. Maduell, Jr., New Orleans Marriage Contracts, 1804-1820 (New Orleans, 1977), a répertorié la plupart des contrats.
} 
pour les gens de couleur libres ${ }^{21}$. Comme nous venons de le voir, ces deux sous-marchés sont caractérisés par des rapports hommes-femmes inverses. Les femmes de couleur dépassent en nombre les hommes de leur race de façon aussi marquée que les hommes blancs l'emportent sur les femmes blanches.

D'ailleurs, les différences sociales entre les races incitent à les considérer comme deux sous-marchés distincts. Contrairement à l'opinion selon laquelle les mulâtres et les noirs libres jouissaient d'un statut privilégié en Louisiane ${ }^{22}$, ceux qui faisaient rédiger des contrats de mariage à la Nouvelle-Orléans - et ils ne sont probablement pas les plus démunis de leur caste - ont déclaré des fortunes nettement inférieures à celles des blancs (tableau 3). Si un contrat existe pour 18\% des alliances des gens de couleur à l'intérieur de l'Église catholique, soit la même proportion que pour les Créoles blancs, la nuptialité diffère cependant beaucoup d'une race à l'autre. Dans les années 1790, quand il est possible de faire des calculs, le taux brut de nuptialité des gens de couleur libres - 3,3 pour 1000 - est inférieur à la moitié de celui des blancs 8,4 pour 1000 - et il n'y a aucune indication de changement au début du $19 \mathrm{e}$ siècle ${ }^{23}$.

TABLEAU 3

Apports au mariage selon le sexe et la race, la Nouvelle-Orléans, 1804-1819

\begin{tabular}{|lcccccr|}
\hline \multicolumn{2}{c}{ hommes } & \multicolumn{4}{c|}{ femmes } \\
\hline \multicolumn{1}{|c}{ race } & moyenne & écart-type & N & moyenne & écart-type & N \\
\hline & $\$$ & $\$$ & & $\$$ & $\$$ & \\
blancs & 8367 & 17218 & 243 & 3945 & 8996 & 299 \\
g.c.l. & 1599 & 1975 & 27 & 1417 & 1641 & 34 \\
total & 7690 & 16469 & 270 & 3687 & 8573 & 333 \\
\hline
\end{tabular}

Source: Archives notariales, Civil District Court, la Nouvelle-Orléans, contrats de mariage, 1804 1819.

21 Article 8 du Digeste du code civil de 1808, 24-25, a interdit explicitement le mariage entre blancs et gens de couleur libre. Voir le Compiled Edition of the Civil Codes of Louisiana (Baton Rouge, La., 1940), vol. 3, partie I, 54.

22 C'est la thèse d'Ira Berlin, Slaves Without Masters; The Free Negro in the Antebellum South (New York, Vintage Books, 1976), chapitre 4, «The Free People of Color of Louisiana and the Gulf Ports», 108-32.

${ }_{23}$ Le taux brut de nuptialité, c'est-à-dire le rapport du nombre des mariages à la population dans une année, se calcule pour les blancs et les gens de couleur à partir des registres du mariage de la cathédrale St-Louis, la seule église où les mariages étaient célébrés dans les années 1790, et des trois recensements de la population de la Nouvelle-Orléans en 1791, 1796, et 1797. Il s'agit dans le texte du taux moyen de nuptialité pour les deux races dans ces trois années. Il n'est plus possible de calculer les taux après 1800 à cause de la proportion de plus en plus importante des Protestants blancs et de couleur dont on ignore le nombre de mariages par année. Par rapport aux gens de couleur libres - catholiques et protestants réunis - recensés en 1820 et 1830 , le nombre de mariages uniquement des catholiques de couleur libres s'élève à 2,79 et 3,21 pour 1000 habitants. En supposant que la grande majorité des gens de couleur continuaient d'être catholiques, même si le taux de nuptialité était en réalité un peu plus élevé, il n'aurait pas été aussi élevé que celui de la population blanche. Voir Paul Lachance, «Intermarriage and French Cultural Persistence in Late Spanish and Early American New Orleans», Histoire sociale/Social History, 15 (mai 1982): 68-69. 
Le surplus féminin chez les gens de couleur libres aurait dû se traduire par des comportements opposés à ceux qui résultent du surplus masculin chez les blancs. A cause de la limitation des effectifs - il n'y a que 32 contrats de mariage entre gens de couleur - les statistiques qui les concernent sont sujettes à caution. Néanmoins, les contrastes raciaux fournissent une perspective comparative interne qui, ajoutée aux comparaisons externes avec la Nouvelle-France et avec Saint-Domingue, permettra de mieux discerner les effets du déséquilibre des sexes sur le fonctionnement du marché matrimonial.

\section{L'influence du déséquilibre des sexes sur l'âge au mariage}

Le déséquilibre des sexes devrait, en principe, favoriser des comportements aptes à rétablir un rapport d'égalité sur le marché matrimonial. Ou bien le mariage retardé du sexe en surplus réduirait le nombre des partenaires possibles de l'autre sexe, ou bien le mariage précoce du sexe sous-représenté augmenterait ses effectifs. En effet, quand le rapport de masculinité en Nouvelle-France est le plus éloigné de l'équilibre, l'âge moyen au mariage est de 28 ans pour les hommes et de 15 ans et demi pour les femmes. Au fur et à mesure que le déséquilibre s'atténue, il y a un léger avancement de l'âge au mariage masculin, qui passe à 27 ans en moyenne, et un recul plus important chez les femmes, qui attendent en moyenne à 23 ans en $1710^{24}$.

Dans les trois paroisses de Saint-Domingue étudiées par Jacques Houdaille, où le rapport de masculinité est beaucoup plus élevé qu'en Nouvelle-France et devient même plus inégal encore à partir de 1770 , l'âge moyen au mariage des hommes blancs monte de 31 ans avant 1760 à 34,1 ans par la suite. Contrairement à ce que prédit le modèle en situation d'accroissement du rapport de masculinité, l'âge des épouses blanches augmente aussi, de 19,7 ans à 21,1 ans, et diffère très peu de l'âge au mariage des femmes de couleur. Par contre, les hommes de couleur se marient cinq ans plus tôt en moyenne que les époux blancs,

\footnotetext{
24 R. Roy et H. Charbonneau, «Nuptialité», op. cit., 292, voir figure 4; Robert Wells, «Quaker Marriage Patterns in a Colonial Perspective», William and Mary Quarterly, $3^{\mathrm{e}}$ sér., 29 (1972): tableau 8, 429 et 431-34. Wells remarque la stabilité relative de l'âge au mariage des hommes qui ne diffère pas non plus de la norme européenne. Les situations de déséquilibre des sexes qu'il cite affectent beaucoup plus l'âge au mariage des femmes et, comme en NouvelleFrance, au fur et à mesure que l'on remonte dans le temps, elles se marient à un âge de plus en plus avancé. Pour expliquer le changement il fait référence aussi à la réduction du déséquilibre des sexes caractérisant la première étape de colonisation: «Migration into a particular colony, whether from Europe or from another colony, tended to be predominantly male. As long as immigrants were an important part of the population, men were likely to be more numerous with the result that at any given age there were far more possible husbands than wives. In order for all the men to marry who wished to do so, it was necessary for them to take wives of a different, and generally younger, age, once most of the eligible women of their own age had married.»
} 
ce qui entraîne comme conséquence que l'écart d'âge entre conjoints est moins grand dans la population de couleur ${ }^{25}$.

On s'attendrait, alors, à ce que les hommes blancs de la NouvelleOrléans se marient aussi à un âge relativement avancé et les femmes blanches très jeunes. Sans que la situation soit exactement contraire dans la population de couleur, la différence entre l'âge au mariage des partenaires devrait être moins grande que dans la population blanche. L'âge exact à la veille du mariage a été déterminé pour 86 maris blancs, 71 femmes blanches, 8 maris de couleur et 8 femmes de couleur ${ }^{26}$. Le tableau 4 en donne la moyenne, la médiane et l'écart-type pour les hommes et les femmes de chaque race, en distinguant les premiers mariages des remariages.

L'excédent des hommes blancs se traduit, d'une part, par un retard de quatre ans au premier mariage sur les hommes de couleur libres et, d'autre part, par le choix d'épouses plus jeunes de quatre ans en moyenne que celles choisies par les hommes de couleur. L'écart d'âge moyen entre les époux de couleur en premières noces est de 3 ans seulement, alors qu'il atteint 11 ans entre les conjoints blancs. L'âge médian au mariage correspond à l'âge moyen pour le sexe masculin des deux races. Le seul résultat inattendu, et il ressort de 8 cas seulement, est la médiane de 18,5 ans chez les femmes de couleur libres, légèrement inférieure à celle de 19,7 ans chez les femmes blanches.

Entre l'âge moyen plus élevé et l'âge médian moins élevé des femmes de couleur, l'âge légal - une indication de l'âge au mariage qui se trouve plus régulièrement dans les contrats - appuie la première mesure de tendance centrale, celle qui suggère que les hommes, qui n'hésitent pas à chercher parmi les filles en très bas âge les épouses qui leur font défaut, sont moins aptes à agir de cette façon dans une population où les femmes sont en surplus. Le code civil de 1808 a fixé à 21 ans l'âge de la majorité pour les deux sexes ${ }^{27}$. Le tableau 5 montre la proportion des mineurs et des adultes de chaque race au moment du premier mariage. Il fait nettement ressortir le jeune âge auquel la plupart des femmes blanches se mariaient. Bien au-delà de la moitié ont contracté

\footnotetext{
25 Jacques Houdaille, «Trois paroisses de Saint-Domingue au XVIIIe siècle: étude démographique», Population, 18, 1 (janvier-mars 1963): 99.

26 Quelques contrats de mariage donnent l'âge chronologique des conjoints. Dans certains cas où les informations manquaient dans les contrats, les inscriptions sur pierres tombales, dont un fichier existe dans le Louisiana State Museum, les certificats de décès dans les archives de la ville de la Nouvelle-Orléans situées à la bibliothèque municipale, et les généalogies publiées ont permis de déterminer l'âge au mariage.

${ }_{27}$ Civil Codes of Louisiana, op . cit., article 19 du Digeste du code civil de 1808, 10-11; article 6, 24-25, qui défend absolument les mariages des garçons au-dessous de 14 ans et des filles au-dessous de 12 ans. Aujourd'hui l'âge minimum du mariage est établi à 18 et à 16 ans respectivement.
} 
leur premier mariage avant d'avoir 21 ans $^{28}$. Beaucoup de femmes de couleur libres se sont aussi mariées avant l'âge adulte, mais dans un pourcentage moins élevé.

A première vue, le mariage de treize blancs mais d'aucun homme de couleur avant leur majorité ne s'accorde pas avec les statistiques de l'âge au mariage pour le sexe masculin des deux races. En fait, le mariage des garçons blancs mineurs est un phénomène exceptionnel. La fortune moyenne déclarée dans les contrats de mariage des mineurs se situe à $23622 \$$, trois fois plus que la moyenne de l'ensemble des maris blancs (voir le tableau 3). Le mariage précoce à la NouvelleOrléans fut un privilège réservé aux fils de riches dont les hommes de couleur libres étaient tout à fait exclus. Et en tant que phénomène exceptionnel, les quelques maris mineurs ne sauraient pas masquer l'âge moyen et médian relativement avancé de l'ensemble des maris blancs.

Une autre indication de l'âge au mariage donnée par les contrats est la mortalité des parents. Lorsque le père et la mère sont présents à la rédaction du contrat, ils y apposent leurs signatures ou leurs marques. Quand lui ou elle est décédé, le notaire met le mot «feu(e)» avant son nom en spécifiant la famille d'origine du contractant. Plus l'époux est âgé, plus forte est la probabilité que son père ou sa mère soit décédé. En effet, $68,7 \%$ des maris blancs sont orphelins de père et $54,2 \%$ sont

\section{TABLEAU 4}

Âge au mariage selon le sexe et la race,

la Nouvelle-Orléans, 1804-1819

\begin{tabular}{|c|c|c|c|c|c|c|c|c|}
\hline & \multicolumn{3}{|c|}{ hommes } & \multicolumn{5}{|c|}{ femmes } \\
\hline race & moyenne & médiane & $\begin{array}{l}\text { écart- } \\
\text { type }\end{array}$ & $\mathrm{N}$ & moyenne & médiane & $\begin{array}{l}\text { écart- } \\
\text { type }\end{array}$ & $\mathrm{N}$ \\
\hline \multicolumn{9}{|c|}{ A. Premiers mariages: } \\
\hline blancs & 32,0 & 30,8 & 9,1 & 76 & 21,0 & 19,7 & 5,8 & 60 \\
\hline g.c.l. & 28,4 & 27,0 & 5,5 & 7 & 25,4 & 18,5 & 11,8 & 8 \\
\hline \multicolumn{9}{|c|}{ B. Remariages: } \\
\hline blancs & 44,5 & 43,5 & 13,8 & 10 & 32,7 & 31,0 & 9,5 & 11 \\
\hline g.c.l. & 41,0 & 41,0 & - & 1 & - & - & - & \\
\hline \multicolumn{9}{|c|}{ C. Tous les mariages: } \\
\hline blancs & 33,5 & 31,8 & 10,5 & 86 & 22,8 & 20,6 & 7,7 & 71 \\
\hline g.c.l. & 30,0 & 27,5 & 6,8 & 8 & 25,4 & 18,5 & 11,8 & 8 \\
\hline
\end{tabular}

Source: Archives notariales, Civil District Court, la Nouvelle-Orléans, contrats de mariage, 18041819 , et diverses sources généalogiques utilisées pour établir l'âge chronologique des contractants. Voir notes 20 et 26.

\footnotetext{
28 La proportion de $59,1 \%$ de mineures parmi les femmes blanches au premier mariage est plus proche de celle observée en Nouvelle-France (64\%) par Y. Landry et J. Légaré, op. cit., 13, en situation du déséquilibre des sexes, que de la proportion des femmes Quakers mineures $(45,4 \%)$ dans la situation plus équilibrée décrite dans R. Wells, «Quaker Marriage Patterns», op. cit., 418.
} 
TABLEAU 5

Âge légal au premier mariage selon le sexe et la race, Nouvelle-Orléans, 1804-1819

\begin{tabular}{|c|c|c|c|c|c|c|c|c|}
\hline \multirow[b]{3}{*}{ âge légal } & \multicolumn{4}{|c|}{ blancs } & \multicolumn{4}{|c|}{ g.c.1. } \\
\hline & \multicolumn{2}{|c|}{ hommes } & \multicolumn{2}{|c|}{ femmes } & \multicolumn{2}{|c|}{ hommes } & \multicolumn{2}{|c|}{ femmes } \\
\hline & $\%$ & $\mathrm{~N}$ & $\%$ & $\mathrm{~N}$ & $\%$ & $\mathrm{~N}$ & $\%$ & $\mathrm{~N}$ \\
\hline mineur(e) & 5,4 & 13 & 58,4 & 128 & - & 0 & 45,5 & 10 \\
\hline adult(e) & 95,6 & 229 & 41,6 & 91 & 100,0 & 19 & 54,5 & 12 \\
\hline total & 100,0 & 242 & 100,0 & 219 & 100,0 & 19 & 100,0 & 22 \\
\hline
\end{tabular}

Source: voir tableau 3.

orphelins de mère, mais seulement $53,7 \%$ et $34,2 \%$ respectivement chez les femmes blanches. D'ailleurs, la survie de plus de mères que de pères témoigne d'un écart d'âge au mariage considérable dans la génération précédente aussi.

La proportion des orphelins de père et de mère chez les hommes de couleur libres, et celle des orphelines de père chez les femmes de couleur libres, s'écartent de $2 \%$ au maximum des proportions correspondantes dans la population blanche. Une différence raciale sensible ne s'observe que dans le pourcentage moins élevé des épouses de couleur qui sont orphelines de mère $(21,4 \%$ contre $34,2 \%$ pour les femmes blanches), ce qui va à l'encontre de l'âge moyen au mariage plus avancé et du plus fort pourcentage de femmes de couleur qui se marient adultes. Le résultat pourrait refléter l'importance de l'autorité maternelle dans les familles de couleur, mais il n'est pas possible de vérifier ici une telle hypothèse. Finalement, s'il y avait une différence ou non dans l'âge au mariage des femmes des deux races, les hommes blancs se marient plus tard que les hommes de couleur libres selon tous les indicateurs et la différence entre l'âge au mariage des partenaires est plus grande dans la population blanche. A ces égards le comportement matrimonial à la Nouvelle-Orléans se conforme bien à celui que laisse prévoir le rapport de masculinité renversé dans les deux races.

\section{Le remariage dans un contexte de déséquilibre}

Le mécanisme de compensation du déséquilibre des sexes devrait se manifester aussi dans les remariages. Au colloque de la Commission internationale de démographie historique de 1979 sur le thème du remariage, les communications portant sur l'Europe ont presque toutes montré une proportion plus grande de veufs que de veuves parmi l'ensemble des mariés ${ }^{29}$. Dans les Amériques, comme nous avons déjà remarqué,

${ }^{29}$ L'étude de Carlo Corsini dans J. Dupâquier, Mariage et remariage, op. cit., 385-396, s'intitule, «Why is remarriage a male affair?», comme si c'était universellement le cas. 
c'était exactement le contraire. Herbert Moller compte dans toutes les colonies de l'Ancien Régime plus de veuves que de veufs participant avec succès au marché matrimonial, lequel comportement est lié explicitement au fait que les femmes sont en pénurie dans le Nouveau Monde tandis qu'elles sont en surplus en Europe ${ }^{30}$.

En Nouvelle-France, à titre d'exemple, les veuves l'emportent en nombre sur les veufs au $17 \mathrm{e}$ siècle alors qu'au siècle suivant, quand les effectifs masculins et féminins approchent de l'égalité, les unions entre veufs et femmes célibataires deviennent plus nombreuses que celles entre maris célibataires et veuves ${ }^{31}$. Que la rareté relative des femmes au commencement de la colonisation ait favorisé le remariage des veuves ressort du fait qu'elles avaient 34,5 ans en moyenne et leur deuxième mari 32 ans seulement ${ }^{32}$.

Sur l'île de Saint-Domingue, la durée du veuvage pour les femmes - 2,7 ans en moyenne - est deux fois moindre qu'en France. Le remariage des veuves est aussi plus important dans la race où les femmes manquent. Houdaille observe que $19 \%$ des épouses blanches sont veuves, mais seulement 7,6\% des femmes de couleur ${ }^{33}$. Ses sources ne lui permettent pas de comparer le remariage des veufs des deux races. A la Nouvelle-Orléans, où le calcul est possible, les rapports inverses de masculinité parmi les blancs et les gens de couleur libres vont de pair avec la fréquence relative du remariage chez les hommes et les femmes (tableau 6). Une femme blanche sur cinq signant un contrat était veuve, contre un mari sur dix qui était veuf. En revanche, un homme de couleur libre sur cinq contractait un remariage et moins d'une femme sur dix. D'ailleurs, toutes les veuves de couleur se remariaient à un veuf tandis que les veuves blanches se remariaient beaucoup plus souvent avec des célibataires qu'avec des veufs. Tel que prévu

TABLEAU 6

État-civil des conjoints selon la race, la Nouvelle-Orléans, 1804-1819

\begin{tabular}{|lcccc|}
\hline & $\begin{array}{c}\text { célibataire/ } \\
\text { célibataire } \\
(\%)\end{array}$ & $\begin{array}{c}\text { veuf/ } \\
\text { célibataire } \\
(\%)\end{array}$ & $\begin{array}{c}\text { célibataire/ } \\
\text { veuve } \\
(\%)\end{array}$ & $\begin{array}{c}\text { veuf/ } \\
\text { veuve } \\
(\%)\end{array}$ \\
\hline blancs $(\mathrm{N}=340)$ & 72,1 & 7,1 & 17,4 & 3.5 \\
g.c.l. $(\mathrm{N}=36)$ & 80,6 & 11,1 & - & 8,3 \\
\hline
\end{tabular}

Source: voir tableau 3.

30 H. Moller, op. cit., 140: «The frequency of remarrying widows - quite contrary to the contemporary European usage - may well be looked upon as successive polyandry in exact contradistinction to the successive polygyny as revealed by European statistics of the age.»

31 Voir surtout le tableau 2 dans H. Charbonneau, «Remariage et fécondité», op. cit., 563.

32 L. Dechêne, op. cit., 109.

33 J. Houdaille, «Trois paroisses», op. cit., 99-100. 
dans le modèle du marché matrimonial, quand les femmes sont moins nombreuses que les hommes, la situation semble plus propice à leur remariage, et vice versa quand elles sont sur-représentées.

La proportion plus élevée des veuves blanches qui se remarient ne signifie toutefois pas qu'elles étaient plus nombreuses à se remarier que les hommes. Dans les contrats de mariage on ne voit que les veufs et les veuves qui réussissent à trouver un nouveau partenaire. On ne peut pas tenir compte de la proportion des hommes et des femmes en état de veuvage qui ne se remarient pas. Étant donné l'écart d'âge entre les conjoints au premier mariage, il est probable que les unions soient brisées beaucoup plus souvent par le décès du mari, jetant par conséquent plus de jeunes veuves de nouveau sur le marché matrimonial ${ }^{34}$.

A en juger par la valeur des fortunes déclarées par les hommes relativement aux dots des femmes, les femmes célibataires avaient la préférence des hommes célibataires de la Nouvelle-Orléans. Il fallait aux veuves une dot plus élevée - $5116 \$$ en moyenne contre $3827 \$$ pour les femmes célibataires - pour obtenir des maris dont les fortunes - 3761 \$ en moyenne - n'arrivaient qu'à un tiers de celles des maris de femmes célibataires ${ }^{35}$. Alors, les femmes qui retenaient de leur premier mariage une certaine fortune avaient de meilleures chances de se remarier, mais cela n'empêche pas que la pénurie de femmes blanches exerçait une influence certaine et positive sur les chances de remariage des veuves. Dans une société où le rapport de masculinité était moins favorable, la fortune exigée pour concurrencer les femmes célibataires aurait été plus élevée, permettant à moins de veuves riches de se remarier.

Donc, dans les grandes lignes, l'influence du déséquilibre des sexes sur le fonctionnement du marché matrimonial à la Nouvelle-Orléans est apparente. L'âge moyen et médian des maris blancs est comparable à l'âge au mariage des hommes de la Nouvelle-France et de SaintDomingue, et il est avancé par rapport à l'âge des hommes de couleur libres dans la même ville. Selon certains indicateurs, les femmes blanches sont en moyenne plus précoces à se marier que les femmes de couleur libres à la Nouvelle-Orléans. Dans les deux races les chances de se remarier varient directement avec la répartition des sexes. Celui qui est en situation de rareté se remarie plus souvent.

Les seuls résultats imprévus sont l'âge médian plus jeune des femmes de couleur et la plus grande proportion de celles-ci qui aient une mère toujours vivante que parmi les femmes blanches. Nous avons

\footnotetext{
34 C'est ce que constatent Y. Landry et J. Légaré, op. cit., 16-17, et Y. Roy et H. Charbonneau, «Nuptialité», op. cit., 293.

35 La fortune moyenne des maris des femmes célibataires est de 9 848\$. Seulement dans les $3,5 \%$ des mariages entre veufs et veuves, celles-ci semblent avoir fait - avec des apports moyens de $1250 \$$ pour des maris dont la fortune moyenne était $7210 \$$ - une alliance financièrement intéressante.
} 
remarqué que ces exceptions sont peut-être liées aux effectifs limités sur lesquels les statistiques concernant la population de couleur sont basées, mais même si l'âge féminin au mariage ne variait pas de façon significative d'une race à l'autre, la différence masculine reste sensible et autorise toute seule à reconnaître l'influence du déséquilibre des sexes sur l'âge au mariage.

\section{Le déséquilibre des sexes et les pratiques exogames}

Il reste à considérer le rapport entre le déséquilibre des sexes et une troisième dimension de la nuptialité: l'endogamie et l'exogamie. Aussitôt que la première génération de Canadiens atteignit l'âge du mariage, et aussi longtemps que des immigrants de France se présentèrent en nombre suffisant sur le marché matrimonial, un système particulier d'intermariage se manifesta en Nouvelle-France. Louise Dechêne décrit les «comportements inattendus» sur l'île de Montréal où au moins un tiers des femmes nées au pays se sont mariées aux immigrants jusqu'en 1715. «Tout se passe comme si les filles préféraient les étrangers», observe-t-elle, «ce qui, économiquement et socialement, est une explication indéfendable» ${ }^{36}$. Dans une étude sur les familles fondées avant 1700 dans trois paroisses rurales près de la ville de Québec, Yves Landry et Jacques Légaré comptent aussi une fille sur trois à se marier aux immigrants de France; pour leur part, plus de la moitié des Français se sont mariés aux Canadiennes. Par contre, aucun homme né au pays ne s'est lié à une femme née dans la métropole, ni évidemment vice versa $^{37}$.

L'indice d'homogamie, c'est-à-dire la fréquence réelle comparée au nombre attendu des unions entre hommes et femmes de la même origine étant donné les rapports de masculinité et les effectifs des groupes représentés sur le marché matrimonial, permet de contrôler le poids des facteurs entraînant inévitablement un certain nombre des mariages exogames. Il montre mieux que les simples pourcentages les préférences réelles des époux d'origines différentes. L'indice d'homogamie varie considérablement entre les paroisses rurales de la Nouvelle-France, où il s'élève à 0,599 , et de l'île de Montréal, où il n'atteint que 0,170 pour les années 1690-1715 $5^{38}$. Dans les deux cas cependant, les Canadiens se marient aux Canadiennes et les Français aux Françaises plus qu'ils ne

\footnotetext{
36 L. Dechêne, op. cit., 102.

37 Y. Landry et J. Légaré, op. cit., 11.

38 L'indice d'homogamie a été proposé et expliqué par Franco Savorgnan dans «Matrimonial Selection and the Amalgamation of Heterogeneous Groups», Population Studies, 3 (mars 1950, supplément): 59-67. Voir la discussion des mesures d'endogamie dans P. Lachance, op. cit., 60-62. L'indice d'homogamie cité ci-dessus pour les paroisses rurales près de Québec est calculé sur les données dans le tableau 2 de Y. Landry et J. Légaré, op. cit., 11, et celui pour Montréal se base sur les données pour les mariages entre Canadiens et Français (c'est-à-dire en éliminant des calculs les quelques conjoints nés dans les colonies anglaises, les Indiens et les inconnus) dans le tableau 8 de L. Dechêne, op. cit., 103.
} 
l'auraient fait dans un assortiment au hasard, ce qui reflète l'identité distincte associée au lieu de naissance presque dès les débuts de la colonisation. En même temps, les alliances relativement nombreuses de Français avec des Canadiennes témoignent d'une interaction entre métropolitains et coloniaux qui s'étend jusqu'à la vie conjugale ${ }^{39}$.

Les études de Houdaille ne posent pas la question des intermariages entre Créoles blancs de Saint-Domingue, immigrants originaires de la France et immigrants blancs d'autres pays et colonies. Il établit plutôt le pourcentage des mariages interraciaux, qui reste assez constant de 1710 à 1790 . Le mode est de $17 \%$. En plus des mariages légitimes, il y avait beaucoup d'unions libres entre blancs et femmes de couleur ${ }^{40}$. Houdaille n'hésite pas à associer le mélange des races à la faible proportion des femmes dans la colonie: «Retenons de ces estimations que l'on comptait de 4 à 5 Blancs pour une Blanche à Saint-Domingue, ce qui explique le métissage rapide de la population.» ${ }^{41}$

Il n'est pas possible de compter les unions interraciales à la Nouvelle-Orléans. A cause de l'interdiction de ce type d'intermariage, l'une des parties avait intérêt à cacher son identité ou - plus souvent - l'union prenait la forme légalement admise du concubinage ${ }^{42}$. Par contre, les registres de mariage, qui donnent systématiquement le lieu de naissance des conjoints, permettent une analyse de l'endogamie et de l'exogamie à l'intérieur des catégories raciales. Entre 1790 et 1840 , les indices d'homogamie décennales pour les trois principaux groupes de langue française dans la population blanche - les Créoles originaires de Louisiane, les Français d'outre-mer, et les réfugiés de Saint-Domingue varient de 0,175 à $0,407^{43}$.

Comme en Nouvelle-France les filles du pays furent plus aptes que leurs frères à conclure un mariage avec un étranger. La moitié des Louisianaises blanches contractèrent des unions exogames contre seulement un Louisianais sur dix ${ }^{44}$. L'échantillon des contrats de mariage accentue l'ouverture aux unions exogames. Trois quarts des contractantes créoles et un quart des contractants créoles envisagèrent une union

\footnotetext{
39 Consulter David Heer, «Intermarriage», Harvard Encyclopedia of American Ethnic Groups, éd. Stephen Thernstrom (Cambridge, Harvard University Press, 1980), 513-514, pour une discussion des inférences sur l'identité ethnique à partir de l'endogamie.

40 J. Houdaille, «Trois paroisses», op. cit., 100-101.

41 J. Houdaille, «Population de Saint-Domingue, op. cit., 863.

42 La pratique des registres de mariage séparés pour les blancs et les gens de couleur libres avait la conséquence de rendre rare l'identification des conjoints en termes de race. On peut imaginer certaines femmes de couleur dans le registre des blancs ainsi que des maris blancs, surtout ceux d'humble condition, dans l'autre registre, mais il est presque impossible d'identifier de tels cas.

43 Ibid., 66, tableau 6

44 P. Lachance, op. cit., 58, tableau 2.
} 
avec un étranger ${ }^{45}$. L'indice d'homogamie des Créoles faisant un contrat entre 1804 et 1819 ne s'élève qu'à 0,206 , en comparaison des indices de 0,369 et de 0,255 calculés pour les deux premières décennies du $19 \mathrm{e}$ siècle à partir des registres de mariage.

Les sous-groupes de la population de couleur furent plus endogames. L'indice d'homogamie des Africains mariés à la NouvelleOrléans dans le demi-siècle commençant en 1790 est de 0,718 , l'indice des Créoles de couleur autochtones est de 0,470 , et celui des réfugiés de couleur de Saint-Domingue est de 0,436. Trois quarts des époux de couleur libres étaient nés en Louisiane, et la proportion des femmes de couleur créoles qui choisirent un partenaire de la même origine $(82,3 \%)$ fut presque aussi élevée que celle du sexe masculin $(90,6 \%)^{46}$. Les Louisianais de couleur faisant un contrat de mariage furent endogames à peu près dans les mêmes proportions ${ }^{47}$. Leur indice d'homogamie est de 0,598 .

Comme explication des alliances exogames observées dans les paroisses près de la ville de Québec, Landry et Légaré font référence à l'antériorité des mariages des Françaises. Se présentant sur le marché matrimonial avant que la première génération des Canadiens atteignent l'âge du mariage, elles se sont mariées en conséquence exclusivement avec les Français. Quand les Canadiens commencèrent à chercher des partenaires, il n'y avait plus de Françaises à prendre. Par contre, se mariant plus tôt que leurs frères, les premières épouses canadiennes se trouvaient forcément liées aux étrangers ${ }^{48}$.

Même si le décalage dans le temps des mariages féminins et masculins de la première génération canadienne peut être valable pour une paroisse fondée peu avant l'arrêt de l'immigration de France, elle ne peut pas expliquer la persistance des mariages exogames au-delà d'une génération, comme à Montréal au début du $18 \mathrm{e}$ siècle ${ }^{49}$, ou à la Nouvelle-Orléans au début du $19 \mathrm{e}$ siècle. Alors, il vaut la peine d'examiner l'hypothèse selon laquelle la répartition des sexes dans la population

45 Dans les contrats de 121 sur 163 femmes créoles $(74,2 \%)$ et de 17 sur 59 hommes créoles $(28,8 \%)$, l'autre partie contractante est née en dehors de la Louisiane. L'exogamie est plus accentuée à cause du pourcentage plus grand des immigrants qui se marient avec un contrat. Il y a un contrat de mariage pour $18,2 \%$ des Créoles et $35,8 \%$ des immigrants nommés dans les registres de mariage des blancs de la cathédrale St-Louis et de la chapelle des Ursulines.

46 P. Lachance, op . cit., tableaux 9 et 10, 73-74. L'indice d'homogamie est calculé sur 69 époux d'origine africaine, 1368 d'origine louisianaise, 344 de Saint-Domingue. Les registres de mariages pour la population de couleur entre 1790 et 1840 donnent aussi les noms de 23 époux nés aux États-Unis. Leur indice d'homogamie - 0,265 - est inférieure à celle des autres groupes, mais il ne s'agit que des individus se mariant dans l'Église catholique. Les mariages entre protestants d'origine américaine, probablement beaucoup plus endogames, échappent à nos calculs.

${ }^{47}$ L'endogamie des contractants de couleur nés en Louisiane est $75 \%$ du côté féminin, et $88 \%$ du côté masculin.

48 Y. Landry et J. Légaré, op. cit., 11.

49 C'est ce qu'avoue L. Dechêne, op. cit., 102, en remarquant les «comportements inattendus» des Montréalaises pendant la deuxième vague d'immigration suivant la paix de Ryswick. 
blanche de la Nouvelle-Orléans a contribué à l'exogamie des femmes créoles et des hommes étrangers. En retardant l'âge au mariage des hommes, en avançant celui des femmes, et en favorisant le remariage des veuves, le surplus des effectifs masculins a-t-il augmenté en même temps le nombre de mariages exogames?

La question aurait une réponse positive si, au delà des effets du déséquilibre des sexes, la migration retardait la préparation à l'établissement: en conséquence, les immigrants se marieraient en général plus âgés que les Créoles. A Saint-Domingue les maris nés en France avaient en moyenne 33,2 ans tandis que ceux nés sur l'île n'avaient que 26,9 ans $^{50}$. Dans l'éventualité d'une différence semblable à la NouvelleOrléans, les immigrants seraient sur-représentés du côté masculin, et les Créoles du côté féminin, c'est-à-dire dans les groupes d'âge avec des chances accrues de se marier à cause du déséquilibre des sexes dans la population blanche. Suivant la même logique, le rapport élevé de masculinité favoriserait des mariages entre étrangers et femmes créoles si celles-ci étaient sur-représentées parmi les veuves qui se remariaient.

Le tableau 7 montre en effet que ceux qui sont nés dans les colonies se marient à un âge plus jeune que les métropolitains à la NouvelleOrléans. La proportion des maris qui sont toujours mineurs au moment de leur premier mariage n'est significative que chez les Créoles. Un peu plus qu'un Louisianais sur cinq n'a pas encore 21 ans. Parmi les 199 époux nés à l'étranger, tous sauf trois ont 21 ans ou davantage. En plus de se marier plus souvent comme mineurs, les Créoles se marient comme adultes plus jeunes que les immigrants. La majorité des maris créoles adultes ont moins de 30 ans, tarıdis que la plupart des immigrants adultes dépassent la trentaine. Dans la mesure où le déséquilibre des sexes aurait découragé le mariage de jeunes hommes - afin de réduire l'offre du sexe excédentaire - il touchait surtout au nombre d'hommes

TABLEAU 7

Âge légal au mariage selon le sexe et l'origine des époux blancs, la Nouvelle-Orléans, 1804-1819

\begin{tabular}{|lrrrrrrrrr|}
\hline & \multicolumn{4}{c}{ Hommes } & \multicolumn{3}{c|}{ Femmes } \\
\hline & \multicolumn{3}{c}{ Mineurs } & \multicolumn{2}{c|}{ Adultes } & \multicolumn{2}{c|}{ Mineures } & \multicolumn{2}{c|}{ Adultes } \\
Origine & $\%$ & $\mathrm{~N}$ & $\%$ & $\mathrm{~N}$ & $\%$ & $\mathrm{~N}$ & $\%$ & $\mathrm{~N}$ \\
\hline Créoles & 23,3 & 10 & 76,7 & 33 & & 62,6 & 72 & 37,4 & 43 \\
Immigrants & 1,5 & 3 & 98,5 & 196 & 37,4 & 53 & 46,2 & 48 \\
total & 5,4 & 13 & 94,6 & 229 & & 58,4 & 128 & 41,6 & 91 \\
\hline
\end{tabular}

Source: voir tableau 3.

\footnotetext{
50 J. Houdaille, «Trois paroisses», op. cit., 99.
} 
créoles se présentant sur le marché et, en conséquence, diminuait les chances des jeunes filles créoles de faire des alliances endogames.

Par contre, en encourageant le mariage des filles à un plus bas âge dans le but de fournir plus d'épouses aux hommes en excédent, le déséquilibre des sexes a privilégié les classes d'âge où les femmes créoles étaient sur-représentées par rapport aux femmes étrangères. Cela augmenta à la fois la probabilité des mariages endogames des hommes créoles et celle des mariages exogames des étrangers. Étant donnée la double pression visant à faire avancer l'âge au mariage des hommes et à faire baisser celui des femmes, le rapport de masculinité élevé de la population blanche affecta surtout l'intermariage entre immigrants et femmes créoles. L'écart d'âge moyen pour les mariages entre deux Créoles est de 8,8 ans; entre un immigrant et une femme créole, il est de 13,3 ans. Plus la différence d'âge entre époux est grande, moins il est probable qu'ils soient de la même origine. Le déséquilibre des sexes, en influençant l'âge au mariage, aurait ainsi accentué la fréquence des mariages exogames à la Nouvelle-Orléans.

Dans la population de couleur aussi, les Créoles semblent se marier plus jeunes que les immigrants. Les trois hommes de couleur libres nés en Louisiane dont l'âge chronologique est connu se sont tous mariés entre 21 et 30 ans, tandis que trois des cinq nés à l'étranger se sont mariés après 30 ans. Trois femmes de couleur sur cinq nées en Louisiane ont moins de 21 ans, alors que la proportion n'est que d'une sur quatre nées à Saint-Domingue. Si le plus jeune âge des Créoles de couleur au mariage n'a pas grossi le nombre des mariages exogames autant que dans la population blanche, la raison se trouve peut-être dans leur rapport de masculinité inverse. Bien que le petit nombre des cas ne permette pas de généraliser avec certitude, la conséquence de la répartition des sexes caractéristique de la population de couleur serait logiquement de rendre moins fréquent chez elle le type d'union - mari âgé et jeune femme - le plus apte à être exogame.

L'autre conséquence démographique du déséquilibre des sexes ne confirme cependant pas l'hypothèse qu'il y ait un lien possible entre la répartition des sexes et la fréquence des alliances endogames et exogames à la Nouvelle-Orléans. Le remariage est avant tout un phénomène des immigrants (tableau 8 ). Le pourcentage plus élevé des immigrants qui se sont remariés est sans doute lié à la structure d'âge de la population blanche. Plus âgés que les Créoles au moment de rédiger leurs contrats, il est aussi plus probable que les immigrants soient veufs ou veuves. Par conséquent, la position avantageuse des veuves dans un marché caractérisé par une pénurie de femmes n'a pas beaucoup ajouté au nombre des mariages entre immigrants et Créoles. Malgré leur âge relativement avancé, 92,5\% des étrangers mariés aux Créoles sont eux aussi célibataires au moment de rédiger leur contrat de mariage. Les 
TABLEAU 8

État civil des époux blancs selon le sexe et l'origine, la Nouvelle-Orléans, 1804-1819

\begin{tabular}{|c|c|c|c|c|c|c|c|c|}
\hline \multirow[b]{3}{*}{ origine } & \multicolumn{4}{|c|}{ hommes } & \multicolumn{4}{|c|}{ femmes } \\
\hline & \multicolumn{2}{|c|}{$1^{\mathrm{er}}$ mariage } & \multicolumn{2}{|c|}{ remariage } & \multicolumn{2}{|c|}{$1^{\text {er }}$ mariage } & \multicolumn{2}{|c|}{ remariage } \\
\hline & $\mathrm{N}$ & $\%$ & $\mathrm{~N}$ & $\%$ & $\mathrm{~N}$ & $\%$ & $\mathbf{N}$ & $\%$ \\
\hline $\begin{array}{l}\text { Créoles } \\
\text { immigrants }\end{array}$ & $\begin{array}{r}54 \\
245\end{array}$ & $\begin{array}{l}91,5 \\
88,4\end{array}$ & $\begin{array}{r}5 \\
32\end{array}$ & $\begin{array}{r}8,5 \\
11,6\end{array}$ & $\begin{array}{l}146 \\
118\end{array}$ & $\begin{array}{l}89,6 \\
69,0\end{array}$ & $\begin{array}{l}17 \\
53\end{array}$ & $\begin{array}{l}10,4 \\
31,0\end{array}$ \\
\hline total & 299 & 89,0 & 37 & 11,0 & 264 & 79,0 & 70 & 21,0 \\
\hline
\end{tabular}

Source: voir tableau 3.

veuves sont le plus souvent immigrantes, et elles se remarient le plus souvent - presque les deux tiers du temps - à un autre immigrant.

En fin de compte, le déséquilibre des sexes a eu une influence contradictoire sur le choix des partenaires. La structure d'âge associée au surplus d'hommes blancs a favorisé des unions endogames de la part des Louisianais et exogames de la part des Louisianaises, mais le remariage a servi plutôt à augmenter le nombre des unions entre immigrants eux-mêmes. Il n'est pas nécessaire de rejeter tout-à-fait l'hypothèse selon laquelle l'intermariage s'explique en partie par l'offre et la demande des sexes au sein de groupes ethniques se faisant concurrence sur le marché matrimonial. La structure d'âge a sûrement pesé sur le choix d'un conjoint plus que la possibilité de remariage. Néanmoins, les résultats nous empêchent d'attribuer le système des alliances entre groupes ethniques exclusivement à la répartition des sexes.

La prudence est d'autant plus exigée que les facteurs socio-économiques ont exercé une influence évidente sur la sélection matrimoniale. C'est finalement à ce niveau d'interprétation qu'a recours Louise Dechêne quand elle remarque que, dans plusieurs cas de mariage précoce des jeunes filles à Montréal, les «parents profitent de la rareté des filles pour en tirer certains avantages sociaux et économiques», et qu'elle constate que, quatre fois sur cinq, l'immigrant qui épouse une veuve "s'épargne 15 ans de défrichements et de difficultés» 51 . Il ne s'agit plus de défrichements à la Nouvelle-Orléans au début du $19 \mathrm{e}$ siècle. Les propriétaires des habitations voisines de la ville s'occupaient plutôt de leur conversion en banlieues, et les immigrants qui devenaient gendres des familles créoles étaient pour la plupart négociants et professionnels ${ }^{52}$. Si les participants au marché matrimonial de la ville faisaient leur choix en vue des avantages sociaux et matériels différents de ceux en vigueur

\footnotetext{
51 L. Dechêne, op. cit., 109.

52 Bien que $38,5 \%$ des contractants créoles soient planteurs ou rentiers, seulement $11,4 \%$ des femmes créoles se marient aux hommes de ces catégories socio-professionnelles. Elles préfèrent plutôt les commerçants $(28,6 \%)$ et les négociants $(20,0 \%)$.
} 
à Montréal au 17e siècle, de telles considérations n'en étaient pas moins importantes.

L'avantage économique des différents types d'alliance varie selon le sexe et l'origine du conjoint. Le tableau 9 montre ce que les Créoles et les étrangers blancs de chaque sexe ont gagné en se mariant à l'intérieur et à l'extérieur de leur groupe. Avec des fortunes moins grandes, les hommes créoles obtiennent des femmes plus riches quand ils se marient à une autre Créole. Inversement, les femmes créoles se marient avec des dots moins importantes aux hommes plus riches en acceptant de faire partie d'une union exogame. S'agit-il d'une coïncidence que l'avantage économique d'un choix endogame pour un Louisianais et son désavantage relatif pour une Louisianaise aillent de pair avec l'endogamie plus forte du sexe masculin?

Les immigrants apportent beaucoup plus au mariage avec une Créole qu'au mariage avec d'autres immigrantes, mais ils sont amplement récompensés par les dots plus grosses des femmes créoles. Quant aux femmes étrangères, quelle que soit l'origine du mari, elles se marient avec des dots à peu près équivalentes et modestes. Les alliances les plus intéressantes sur le plan économique, celles où le mari est Créole, sont exceptionnelles. Dans ce cas-ci, ce qui fut dans l'intérêt de la femme ne profita pas autant au mari, à la différence des unions mutuellement avantageuses entre immigrants du sexe masculin et femmes créoles ${ }^{53}$.

TABLEAU 9

Apports moyens des conjoints blancs selon le type de mariage et leur origine, la Nouvelle-Orléans, 1804-1819

\begin{tabular}{|lrrrr|}
\hline & \multicolumn{2}{c}{ hommes } & \multicolumn{2}{c|}{ femmes } \\
\hline $\begin{array}{l}\text { type de mariage et } \\
\text { origines du couple }\end{array}$ & \multicolumn{1}{c}{$\$$} & $\mathrm{~N}$ & $\$$ & $\mathrm{~N}$ \\
\hline $\begin{array}{l}\text { A. Mariages endogames } \\
\text { Créole-Créole }\end{array}$ & 10130 & 36 & 7042 & 37 \\
immigrant-immigrante & 3920 & 45 & 2772 & 62 \\
B. Mariages exogames & 11460 & 11 & 2865 & 15 \\
Créole-immigrante & 11612 & 87 & 4059 & 106 \\
immigrant-Créole & 5558 & 64 & 3466 & 79 \\
immigrant-immigrante & 8367 & 243 & 3945 & 299 \\
total & & & & \\
\hline
\end{tabular}

Source: voir tableau 3.

Note: Le tableau donne la moyenne des apports de tous les époux dans chaque groupe, même quand il n'y a aucune mention des biens par le partenaire.

\footnotetext{
53 Les unions les moins avantageuses pour une immigrante sont celles avec un mari de la même nationalité. Les Françaises, cependant, font exception à cette règle. Elles apportent $3200 \$$ en moyenne aux mariages exogames avec des hommes dont la fortune moyenne se limite à $3271 \$$. A titre de comparaison, dans les mariages avec les Français - et trois quarts des Françaises contractent des mariages endogames - elles contribuent $2832 \$$ et leur mari $3578 \$$.
} 
En conclusion, le grand écart d'âge au mariage et la fréquence exceptionnelle du remariage des veuves dans la population blanche de la Nouvelle-Orléans confirment l'hypothèse des comportements compensateurs résultant du déséquilibre prononcé des sexes. Mais aussi importantes que soient les conséquences démographiques du rapport de masculinité, elles ne constituent qu'une explication partielle des alliances endogames et exogames à la Nouvelle-Orléans. Pour avancer plus loin dans l'analyse de la sélection des époux, il sera nécessaire de prendre en compte d'autres variables, et en particulier les considérations socioéconomiques de ceux et celles qui se trouvent sur le marché matrimonial. 